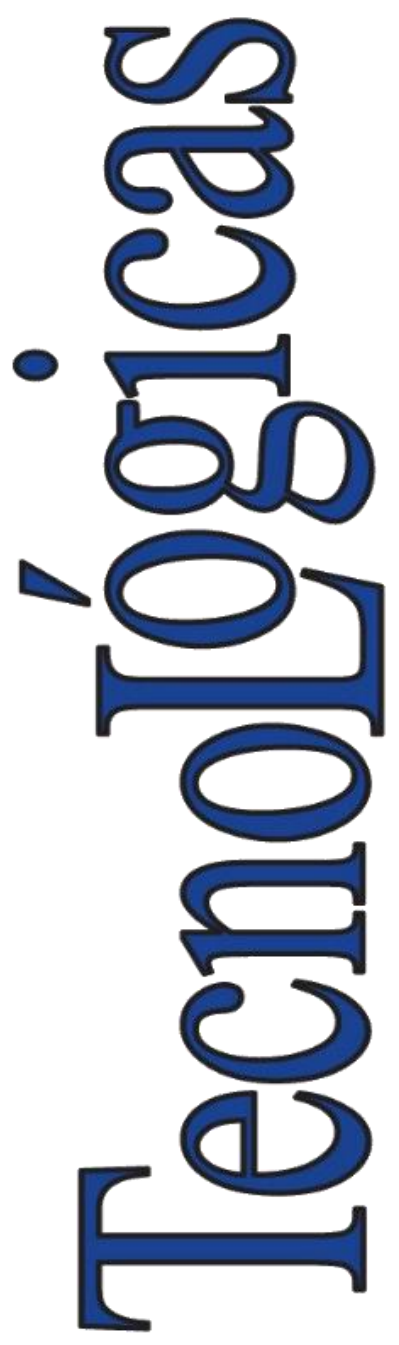

ISSN-p: 0123-7799 ISSN-e: $2256-5337$

Vol. 24, nro. 50, e1701

Recibido: 23 junio 2020 Aceptado: 11 noviembre 2020 Disponible: 17 diciembre 2020

CInstituto Tecnológico Metropolitano Este trabajo está licenciado bajo una Licencia Internacional Creative Commons Atribución (CC BY-NC-SA)

\section{Modelo para la escritura de artículos científicos a distancia mediante tareas colaborativas}

\section{Model for Writing Scientific Articles Remotely Through Collaborative Tasks}

\author{
(D) Andrés Felipe Solis-Pino ${ }^{1}$; \\ (D)Leydi Mercedes Vargas-Ordoñez ${ }^{2}$; \\ (DCésar A. Collazos ${ }^{3}$; \\ 1 Universidad del Cauca, Popayán-Colombia, \\ afsolis@unicauca.edu.co \\ 2 Universidad del Cauca, Popayán-Colombia, \\ leydivargas@unicauca.edu.co \\ ${ }^{3}$ Universidad del Cauca, Popayán-Colombia, \\ ccollazo@unicauca.edu.co
}

\section{Cómo citar / How to cite}

A. F. Solis-Pino; L. M. Vargas-Ordoñez; C. A. Collazos "Modelo para la escritura de artículos científicos a distancia mediante tareas colaborativas", TecnoLógicas, vol. 24, nro. 50, e1701, 2021.

https://doi.org/10.22430/22565337.1701

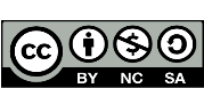




\title{
Resumen
}

Para mejorar el proceso de escritura científica de forma colaborativa se han realizado múltiples investigaciones, como la elaboración de indicadores para medir el éxito de estas actividades y el desarrollo de herramientas informáticas que faciliten la ejecución de dicho ejercicio. Estos trabajos de investigación han tomado relevancia y pertinencia por las condiciones actuales de pandemia (COVID-19), debido a que las medidas de aislamiento general que se han establecido imposibilitan el encuentro y la interacción con otras personas, lo cual son valores importantes para tener éxito en cualquier actividad colaborativa. Por esta razón, en tiempos recientes, el proceso de investigación y escritura científica se ha dificultado, identificándose la falta de un proceso estructurado para organizar y sistematizar la investigación científica a distancia mediante medios virtuales. Teniendo en cuenta la problemática mencionada, se presenta un modelo basado en los aportes de la ingeniería de la colaboración que permite diseñar tareas conjuntas desde la virtualidad, añadiendo elementos del modelo Socialización, Externalización, Combinación e Internalización (SECI), el cual busca gestionar el conocimiento y hacerlo uniforme entre los autores. Para esto, se realizó una encuesta que permitió la abstracción de tareas comunes al escribir artículos científicos y se planteó un modelo para mejorar este proceso. Su validación se realizó mediante un caso de estudio donde un grupo de investigadores utilizan la propuesta y desarrollan la escritura de un documento de investigación. Posteriormente, se obtuvieron resultados prometedores acerca del modelo propuesto, con niveles altos de satisfacción entre los participantes. Finalmente, se constató que es un área de investigación en la que se pueden hacer aportes significativos al proceso de escritura científica en épocas de aislamiento a causa de la pandemia, donde el desarrollo de herramientas específicas para evaluar actividades colaborativas es una necesidad latente.

\section{Palabras clave}

Trabajo colaborativo, modelo SECI, escenarios virtuales, escritura colaborativa, gestión del conocimiento.

\begin{abstract}
There has been a lot of research on improving the process of scientific writing collaboratively, for example, indicators have been elaborated to measure the success of this type of activities, also, computer tools have been developed to facilitate this exercise, among other proposals. These research works have become relevant and pertinent due to the current conditions of pandemic (COVID-19), because, general isolation measures have been established that make it impossible to meet and interact with other people, which are important values to succeed in any collaborative activity. For this reason, the process of scientific research and writing has become extremely difficult in recent times; specifically, the lack of a structured process to organize and systematize scientific research at a distance through virtual means has been identified. Considering the above-mentioned problems, a model based on the contributions of collaborative engineering is presented, which allows the design of joint tasks from virtuality, adding elements of the SECI model, which seeks to manage knowledge and make it uniform among authors. To this end, the abstraction of common tasks when writing scientific articles was carried out and a model was proposed to improve this process. The validation of this was done through a case study where a group of researchers use the proposal and develop the writing of a research paper. Subsequently, promising results were obtained about the proposed model, with high levels of satisfaction among participants. Finally, it was found that this is an area of research in which contributions can be made, where the development of specific tools to evaluate collaborative activities is a latent need.
\end{abstract}

\section{Keywords}

Collaborative work, SECI model, virtual scenarios, scientific writing, knowledge management. 


\section{INTRODUCCIÓN}

El proceso de escritura de artículos científicos es una actividad que tiene un alto grado de complejidad, puesto que existe mucha información que debe ser condensada y estructurada para lograr un texto coherente [1]. Sumado a esto, factores como el grado de formación de los autores, la experiencia en escritura científica, la ubicación geográfica o incluso el tipo de colaboradores son elementos determinantes para el éxito o no de una publicación [2].

Precisamente estos dos últimos factores han tomado relevancia a partir del escenario complicado que afronta el mundo con la Pandemia de COVID-19, debido a que los investigadores han tenido que adaptarse a realizar el trabajo de producción y escritura científica a distancia, convirtiendo esta actividad en un proceso más complejo de lo que ya era; además, existen componentes emocionales, personales y sociales que surgen cuando individuos interactúan entre sí con un objetivo en común: publicar exitosamente el documento; es decir, problemáticas típicas de una organización o agrupación de individuos.

Para resolver estos problemas de distanciamiento geográfico, se han creado herramientas que permiten y facilitan el trabajo a distancia. Específicamente en el área académica y de investigación se han desarrollado procesadores de texto colaborativos, plataformas de educación en línea y medios de comunicación, entre otros [3]. Estos instrumentos forman parte del concepto de Espacio Virtual para el Trabajo Colaborativo (EVTC), que puede definirse como un espacio basado en la conectividad de la web que permite el trabajo colaborativo de grupos en los que sus miembros están físicamente distantes [4]. Actualmente, estas herramientas satisfacen de mejor manera las demandas de trabajo y comunicación entre autores, pero la falta de un modelo o método estructurado que sistematice las actividades requeridas en el proceso de producción científica a distancia es un problema importante que no ha sido explorado.

En la actualidad, una de las problemáticas recurrentes en las organizaciones empresariales (un conjunto de personas con un objetivo en común [5]) tiene que ver con la apropiación del conocimiento organizacional. Este concepto se refiere a cómo las compañías logran que todos sus integrantes constituyan el conocimiento, de tal modo que este sea sistemático y articulado para la empresa, más allá de los individuos que la componen [6]. En este sentido se han desarrollado múltiples teorías y modelos [7], [8] para convertir el conocimiento tácito de las personas en conocimiento para las organizaciones empresariales.

Entre estas propuestas se planteó el modelo SECI, que consiste en una estrategia de creación, generación y transferencia del conocimiento que establece que las experiencias personales, el conocimiento específico y los procedimientos puntuales forman parte de la empresa, y deben ser preservados y articulados para el futuro. En este modelo se pueden distinguir cuatro dimensiones: socialización, externalización, combinación e internalización; además, se añade un concepto denominado "Ba", que puede pensarse como un espacio compartido (no necesariamente un lugar físico) en el que el conocimiento interactúa con los participantes [9].

Teniendo en cuenta que la redacción de artículos científicos es un proceso de transmisión de las experiencias (conocimiento) de los investigadores, se buscó combinar el modelo SECI con el concepto de EVTC, para que de forma implícita el conocimiento pueda estructurarse uniformemente en la organización (en este caso, los realizadores del proceso de producción científica) y alcanzar así sus objetivos o productos finales. Es por esto por lo que este documento propone un modelo colaborativo para la escritura de artículos científicos a distancia con elementos del modelo SECI, para apoyar y definir las actividades de un proceso investigativo y, de esta forma, ayudar a los autores que por las circunstancias actuales de 
aislamiento no pueden reunirse en persona, lo que genera dificultades de coordinación, comunicación y realización de tareas conjuntas.

El modelo contendrá los aportes de la ingeniería de la colaboración que permita diseñar actividades desde la distancia y la virtualidad, y con base en el modelo SECI se busca que este permita gestionar el conocimiento y lograr que el aprendizaje sea estructurado y uniforme [10].

\subsection{Referentes teóricos}

A continuación, se exponen los principales conceptos referentes a la ingeniería de la colaboración y el modelo propuesto.

\subsubsection{Ingeniería de la Colaboración}

La Ingeniería de Colaboración (IC) es un acercamiento al diseño de procesos colaborativos reutilizables [11]. Los procesos colaborativos son esfuerzos entre dos o más personas que buscan alcanzar objetivos mutuos, estos necesitan ser explícitamente diseñados, estructurados y manejados, debido a que son el eje central de esta área de estudio. El documento de Coto y Collazos, en [12], expresa que la ingeniería de la colaboración "es el diseño de procesos repetitivos colaborativos, los cuales se pueden transferir a grupos, usando técnicas y tecnologías". Los patrones de colaboración y los thinklets son una parte esencial de la IC [13].

\subsubsection{Patrones de colaboración}

En las investigaciones realizadas en el campo de la ingeniería de la colaboración, se han establecido una serie de patrones relacionados con la forma en la cual un grupo trabaja conjuntamente hacia sus metas. Estos patrones son métodos basados en la experiencia para usar y reutilizar formas comprobadas de organizar la comunicación y las actividades para resolver tareas grupales [14]. Cada patrón tiene subpatrones que pueden relacionarse con las actividades de la descripción del proceso genérico. Normalmente, los patrones de colaboración pueden clasificarse en: generación, reducción, clarificación, organización, evaluación y construcción de consenso [15].

\subsubsection{Thinklets}

Son las descripciones relacionadas con la forma en que se llevan a cabo las actividades. Los thinklets son interacciones predecibles, repetibles y transferibles que ocurren cuando las personas realizan actividades colaborativas [16]. Un thinklet es la unidad fundamental intelectual necesaria para crear un patrón de colaboración [17]. Estos elementos deben ser completamente detallados y modificables, y pueden usarse para construir nuevos grupos de procesos de colaboración, estos deben ser fácilmente aprendidos, recordados y adaptados [18].

\subsubsection{Escenarios colaborativos virtualizados}

En un escenario colaborativo es fundamental que se garanticen tres elementos clave para una colaboración fluida. En primer lugar, la denominada interdependencia positiva (obligaciones mutuas que contribuyen a un objetivo común); el segundo es la responsabilidad individual; y el tercero es la igual participación [19]. Si estos elementos están presentes en la 
actividad colaborativa permite a cada individuo aprender más de lo que aprendería por sí mismo (conocimiento compartido) [20], que exista reciprocidad entre los miembros para que se construya el conocimiento y que se cree una conciencia de responsabilidad grupal (todos ganan o todos pierden) [21].

Otros elementos que desempeñan un papel importante en las actividades colaborativas son la comunicación, las relaciones simétricas y recíprocas, el tamaño del equipo, las estrategias de colaboración, las técnicas que se empleen en el proceso, los roles de cada miembro y el diagnóstico de la dinámica grupal [22].

Cuando se habla de un escenario colaborativo desde la virtualidad se producen algunas variables; no obstante, a pesar de compartir un espacio común y de realizar encuentros con los otros miembros, las características específicas de este espacio son las que condicionan la forma de relación e interacción del grupo [23].

En el caso de los grupos de trabajo virtuales mediados por la tecnología, el elemento observable se ha de ponderar y registrar sobre otros indicadores que representan los elementos que componen al entorno virtual [24]. Adicionalmente, se debe contar con EVTC con funcionalidades que aporten al proyecto, así cada uno de los miembros del grupo estará informado en tiempo real (awareness) de las actividades que se desarrollen [25]. Por lo tanto, es importante mencionar que el concepto de awareness en espacios virtuales es fundamental, porque permite obtener una percepción del grupo y de sus actividades, lo cual es de gran ayuda para reducir el esfuerzo comunicativo necesario para desarrollar actividades colaborativas. En otras palabras, crea la necesidad de comunicación entre los individuos con el fin de avanzar hacia un objetivo en común [26].

\subsubsection{Modelo SECI}

El modelo SECI es un método de transformación que aboga por la existencia de cuatro formas de transmisión del conocimiento, y que procura que el saber en una organización sea uniforme. A continuación, se mencionan:

Socialización: el conocimiento se comparte mediante interacciones sociales. Un ejemplo en este contexto son las reuniones grupales entre autores, donde se utilizan medios tecnológicos para debatir ideas acerca de cuestiones sobre la producción científica [27].

Externalización: es un proceso que intenta cristalizar el conocimiento a través de medios o ayudas visuales (imágenes, escritos, cuadros u otros). En la investigación, esta forma de transmisión del conocimiento se lleva a cabo en varias actividades colaborativas, como la exposición de resultados y su condensación mediante tablas o figuras [28].

Combinación: esta forma de transmisión del conocimiento se realiza mediante la unión de varios medios de externalización. Está emparentado con la realización de prototipos funcionales [29].

Internalización: convierte el conocimiento explícito a tácito y está directamente relacionado con la metodología learning by doing, el cual menciona que la mejor forma de aprender es llevando a cabo los conocimientos aprendidos [28].

\subsection{Trabajos relacionados}

El artículo de Guerrero et al., en [30], presenta la herramienta CCCuento diseñada para apoyar a grupos de estudiantes en la creación colaborativa de cuentos infantiles, pretendiendo mejorar el uso del lenguaje y el desarrollo de las habilidades de escritura. La herramienta sigue un modelo que incluye aspectos como la interdependencia positiva y la igual participación a través de una secuencia de actividades mezcladas con períodos de descanso 
para su revisión y discusión. CCCuento incorpora la interdependencia positiva al organizar la tarea de escribir cuatro historias de forma simultánea en cinco fases: nombrar la historia, introducción, cuerpo A, cuerpo B y secuencia final. Además, cada participante debe trabajar en todas las fases. En la etapa final de la actividad, el profesor evalúa y califica el trabajo grupal. También existen tareas individuales, por lo que el equipo debe estar coordinado, con objetivos y roles claros, para asegurar el éxito en la actividad; concretamente, cada estudiante es responsable de completar una parte de la historia durante cada fase. Este documento también refleja que un requisito para etiquetar una actividad como colaborativa es tener acción, conocimiento y simetría de estado.

Por otra parte, en el documento de Ubilla Rosales et al, en [31], se pretende determinar el efecto de la escritura colaborativa en el aprendizaje de un idioma extranjero mediante el uso de una herramienta (procesador de texto) en línea, además, de sesiones semipresenciales.

Estos resultados los compara con un grupo de control en el que se utilizaron los mismos criterios de enseñanza, pero sin las actividades colaborativas. Para determinar el nivel de influencia de la colaboración en el proceso de aprendizaje, se utilizan técnicas estadísticas en un diseño cuasiexperimental, en el que se compara un grupo de control y un grupo experimental. La determinación del nivel de inglés se realizó según el examen del marco común europeo [32]; asimismo, para mejorar la fiabilidad de los datos resultantes, se utilizaron cuatro evaluaciones consistentes en un preprueba y tres pospruebas. Entre los principales resultados obtenidos se encontró que los estudiantes que utilizaron la escritura colaborativa demostraron un desempeño mejor en la producción de textos en inglés, en comparación con el grupo de alumnos que trabajaron individualmente. También, se concluyó que las tareas colaborativas ayudan a desarrollar mejores procesos cognitivos que permiten la adopción de una segunda lengua de forma más natural.

En esta línea, el artículo de Ness et al, en [33], expresa la necesidad de organizar grupos para mejorar el proceso de producción de artículos científicos, mediante actividades colaborativas en las ciencias de la salud. En principio se forma un grupo de personas con experiencia en escritura de documentos de investigación, donde se organiza una reunión general que discute temas relacionados con el proyecto, como los objetivos del documento, los intereses, las áreas de conocimiento y las responsabilidades de cada miembro; asimismo, se plantean una serie de tópicos para mejorar el proceso de escritura colaborativa, como la celebración de reuniones regulares, la asignación responsabilidades y las fechas de entrega de las tareas asumidas. Aunque en el documento no se evidencia explícitamente, se puede notar que en las actividades colaborativas existen elementos como la responsabilidad individual y la interdependencia positiva; por lo tanto, son actividades que son modelables y replicables. Es importante mencionar que el documento no proporciona una metodología estructurada para ser replicada, pero presenta resultados como la obtención de tres artículos de investigación y una editorial a partir de las directrices planteadas en el documento.

Finalmente, los autores concluyen que los grupos de redacción colaborativos son una forma de mejorar la producción científica en las ciencias de la salud. Entre las principales herramientas utilizadas se encuentran los procesadores de texto en línea, herramientas de organización de proyectos y chats grupales.

Otro artículo que relaciona el trabajo colaborativo y la escritura de artículos científicos es el de Hasanuddin et al, en [34], donde los autores indagaron sobre la problemática del aprendizaje y el mejoramiento en la redacción de investigaciones, planteando que es un proceso tomado como obligatorio y de forma poco natural, por lo que el autor concluye que el nivel de escritura científica en los estudiantes asiáticos es muy bajo. Para atacar este problema, se propone una metodología para la enseñanza de la escritura científica a través del trabajo colaborativo, utilizando la investigación-acción. En el modelo se proponen cuatro 
etapas (planificación, actuación, observación y reflexión), además de dividir a los estudiantes en grupos para realizar sus tareas, generando así interdependencia positiva. La dinámica propuesta es la siguiente: se asignan una serie de tareas a cada uno de los integrantes del grupo, luego los mismos individuos evalúan el desempeño de sus compañeros y consolidan sus resultados en un documento; por último, el profesor evalúa los resultados y socializa cualquier error. La evaluación del método se realizó mediante un caso de estudio, haciendo un pretest y un postest, Finalmente, se concluye que el trabajo colaborativo permite proporcionar mejor información, perspectivas y opiniones comparado al realizarlo individualmente.

Por otra parte, es importante mencionar que existen investigaciones que permiten viabilizar el proceso de escritura científica a través de estrategias y herramientas. Este es el caso del documento propuesto por Singh y Mayer, en [3], en el cual expresa la dificultad de realizar escritos científicos de calidad; en consecuencia, brinda una serie de estrategias y herramientas que podrían ayudan a mejorar este proceso. En primer lugar, recomienda iniciar la escritura del documento paralelamente al desarrollo de la investigación; además, propone guías existentes en la literatura que pueden mejorar el proceso científico. Asimismo, aconseja que se debe realizar el proceso de investigación de forma colaborativa, ya que es beneficioso para el producto final, debido a que añade nuevas perspectivas, objetivos, argumentos y conclusiones a la investigación; igualmente dice que la retroalimentación de otras personas es beneficiosa para detectar incoherencias, estructuras de texto inapropiadas y mensajes poco claros en el documento. Por último, propone una serie de herramientas que permiten el trabajo colaborativo entre las personas.

Por último, es importante decir que la revisión bibliográfica realizada se llevó a cabo en las bases datos Scopus, IEEE Xplore, ScienceDirect y en Google Académico, utilizando cadenas de búsqueda estructuradas para cada repositorio de investigaciones, arrojando como resultado que existen investigaciones [33], [35], [36] que proponen herramientas y/o metodologías para mejorar el proceso de escritura colaborativa en el ámbito científico, pero que estas no están ideadas para utilizarlos a distancia y mucho menos propuestas con la integración de un modelo de gestión del conocimiento, como es el caso de la presente investigación, por lo tanto, es un vacío en el área de la ingeniería de la colaboración que puede ser trabajado.

\section{METOdOLOGía}

En esta sección se procede a describir el modelo propuesto.

\subsection{Modelo de escritura de artículos científicos a distancia mediante virtualidad}

El modelo colaborativo propuesto denominado GCCWrite se basa en la premisa de que la producción de artículos científicos es un proceso de transmisión del conocimiento, que puede ser modelado y sistematizado. Si bien es cierto que en los documentos de investigación los autores pretenden proporcionar información al lector, también esta información se transmite de autor a autor, por lo que, si el conocimiento es uniforme entre todas las partes, se obtendrán mejores resultados en el artículo redactado; de igual forma se asume que el grupo de investigadores es una organización que trabaja por un objetivo común.

Por lo general, la escritura de artículos de forma colaborativa se realiza mediante la división de tareas, y posteriormente estos resultados se acoplan en un documento. Esto conlleva a dificultades como falta de coherencia, participación inequitativa o la distribución no homogénea del conocimiento entre los autores. Sumado a esto, por el contexto actual 
(virtualidad forzosa), también se añaden problemáticas como la falta de definición de objetivos claros, escasa interdependencia positiva entre los participantes y la falta de comprensión del contexto en el que se trabaja (awareness), que son factores ampliamente aceptados para determinar el éxito de una actividad colaborativa [37]. Es por esto que el modelo propuesto combina criterios de la ingeniería de la colaboración con el modelo de gestión del conocimiento SECI, para resolver la problemática acerca de cómo mejorar el proceso de producción científica a distancia.

La escritura de artículos de investigación es un proceso que se compone de una serie de actividades que se realizan acorde a su ejecución temporal. En el modelo propuesto estas actividades se han encapsulado en fases (preproceso, proceso y postproceso), tomando como referente a Collazos en [38], donde propone dividir la escritura de cuentos colaborativos; pero, en el caso de esta investigación, se adaptó a la escritura de artículos de investigación.

Adicional a esto, se agregó el componente del modelo SECI, utilizando los cuatro modos de conversión del conocimiento, consistente en la socialización (tácito a tácito), externalización (tácito a explícito), combinación (explícito a explícito) e internalización (explícito a tácito), para permitir que el conocimiento sea homogéneo entre los autores. De la misma manera se agregó un último elemento denominado "Ba", el cual se entiende como el lugar donde se realiza la transmisión del conocimiento y se dan las interacciones entre los participantes. En la Figura 1 se puede observar una descripción del modelo GCCWrite con sus componentes principales.

El modelo propuesto (Figura 1) se ha dividido en tres fases denominadas preproceso, proceso y postproceso. Cada una de estas fases está compuesta por una serie de actividades que deben realizarse independientemente del tipo o tema del artículo a redactar. Estas se han seleccionado mediante un proceso de recolección de información, en el que se les preguntó a los investigadores cuáles actividades eran recurrentes (abstracción) en el momento de realizar producción científica de forma colaborativa. Para esto se diseñó una encuesta a 20 personas con experiencia investigativa de distintas nacionalidades (Argentina, Colombia y Perú) de los cuales el 15 tenía más de 7 artículos publicados; el $40 \%$, entre 4 y 6 artículos y el $45 \%$ restantes, entre 1 y 3 documentos de investigación. Sumado a esto, también se les cuestionó por temas concernientes a la producción científica y su opinión sobre un modelo para mejorar este tipo de actividades. Posteriormente se filtraron las actividades propuestas por los investigadores con relación a si estas ameritan realizarse colaborativamente y a distancia.

Para esto, las actividades deben cumplir tres criterios: ser realizada por varias personas, hacerse por personas geográficamente dispersas y que contribuya al objetivo común del grupo [39]. En la Tabla 1 se pueden observar las actividades filtradas según los criterios expuestos anteriormente.

Ahora, una vez obtenidas las actividades genéricas que permiten contribuir a la escritura de documentos científicos, se procede a modelarlas de forma colaborativa. Para esto se estructuraron considerando los tres principios básicos de la colaboración: igual participación, responsabilidad individual e interdependencia positiva [40]. Sumado a esto, las actividades colaborativas fueron modeladas usando thinklets. A continuación, se puede observar un ejemplo de este tipo de modelamiento que, para efectos del presente documento, solo se exponen algunas. 
Tabla 1. Actividades propuestas por los expertos y si estas se pueden interpretar como colaborativas.

Fuente: elaboración propia.

\begin{tabular}{|c|c|c|c|}
\hline Actividades & $\begin{array}{l}\text { Realizada } \\
\text { por varias } \\
\text { personas }\end{array}$ & $\begin{array}{l}\text { Realizada por miembros } \\
\text { geográficamente } \\
\text { dispersos } \\
\end{array}$ & $\begin{array}{l}\text { La actividad } \\
\text { contribuye al } \\
\text { objetivo común }\end{array}$ \\
\hline $\begin{array}{l}\text { Definir temática principal del artículo y subtemas } \\
\text { que pueden aportar a resolver la problemática. }\end{array}$ & $\checkmark$ & ( & $\checkmark$ \\
\hline Definir viabilidad del estudio. & $\checkmark$ & $\checkmark$ & $\checkmark$ \\
\hline $\begin{array}{l}\text { Lluvia de ideas sobre posibles soluciones para la } \\
\text { problemática propuesta. }\end{array}$ & $\checkmark$ & $\checkmark$ & $\checkmark$ \\
\hline $\begin{array}{l}\text { Definir metodología que se va a utilizar para } \\
\text { resolver la problemática. }\end{array}$ & $\checkmark$ & $\checkmark$ & $\checkmark$ \\
\hline Asignar roles a los participantes. & $\checkmark$ & $\checkmark$ & $\vee$ \\
\hline Definir fechas de avances y entrega final. & $\vee$ & $\checkmark$ & $\vee$ \\
\hline $\begin{array}{l}\text { Socializar la problemática con los otros autores, } \\
\text { cuestiones acerca de esta, o del fenómeno de estudio. } \\
\text { Definición del tipo de producción científica a realizar }\end{array}$ & $\checkmark$ & $\checkmark$ & $\checkmark$ \\
\hline $\begin{array}{l}\text { (artículo científico, documento de conferencia, } \\
\text { proyecto, entre otros). }\end{array}$ & $\checkmark$ & $\checkmark$ & $\checkmark$ \\
\hline Definir medios de comunicación entre los autores. & $\checkmark$ & $\checkmark$ & $\checkmark$ \\
\hline $\begin{array}{l}\text { Definición de la forma de presentar los resultados } \\
\text { específicos del artículo científico. }\end{array}$ & $\checkmark$ & $\checkmark$ & $\checkmark$ \\
\hline $\begin{array}{l}\text { Determinar revista o evento en particular para } \\
\text { enviar. }\end{array}$ & $\mathbf{x}$ & $\mathbf{x}$ & $\checkmark$ \\
\hline Desarrollo de la metodología propuesta. & $\checkmark$ & $\checkmark$ & $\vee$ \\
\hline $\begin{array}{l}\text { Búsqueda de antecedentes relacionados con el } \\
\text { fenómeno estudiado. }\end{array}$ & $\checkmark$ & $\checkmark$ & $\checkmark$ \\
\hline $\begin{array}{l}\text { Plasmar sobre papel las experiencias (escritura del } \\
\text { documento). }\end{array}$ & $\checkmark$ & $\checkmark$ & $\checkmark$ \\
\hline $\begin{array}{l}\text { Realización del proceso de experimentación o estudio } \\
\text { de caso (si es necesario). }\end{array}$ & $\checkmark$ & $\checkmark$ & $\checkmark$ \\
\hline Obtención y selección de resultados relevantes. & $\checkmark$ & $\checkmark$ & $\checkmark$ \\
\hline $\begin{array}{l}\text { Reestructuraciones o correcciones al artículo } \\
\text { científico o las metodologías propuestas. }\end{array}$ & $\checkmark$ & $\checkmark$ & $\checkmark$ \\
\hline $\begin{array}{l}\text { Aplicación de una estrategia o técnica estadística a } \\
\text { los resultados. }\end{array}$ & $\checkmark$ & $\checkmark$ & $\checkmark$ \\
\hline $\begin{array}{l}\text { Definición de conclusiones o aportes principales del } \\
\text { trabajo. }\end{array}$ & $\checkmark$ & $\checkmark$ & $\checkmark$ \\
\hline $\begin{array}{l}\text { Realización de las correcciones y comentarios de los } \\
\text { pares académicos. }\end{array}$ & $\checkmark$ & $\checkmark$ & $\checkmark$ \\
\hline $\begin{array}{l}\text { Definición de estrategia para corrección o } \\
\text { sugerencias por parte de los evaluadores. }\end{array}$ & $\checkmark$ & $\checkmark$ & $\checkmark$ \\
\hline $\begin{array}{l}\text { Consultar a una fuente externa para evaluación } \\
\text { conceptual, metodológica o gramatical. }\end{array}$ & $\mathbf{x}$ & $\mathbf{x}$ & $\checkmark$ \\
\hline Publicación del artículo en modo preimpresión. & $\mathbf{x}$ & $\mathbf{x}$ & $\checkmark$ \\
\hline Envió a revisión por pares. & $\vee$ & $\checkmark$ & $\checkmark$ \\
\hline Aceptación o no de las sugerencias por pares. & $\checkmark$ & $\checkmark$ & $\vee$ \\
\hline $\begin{array}{l}\text { Proceso de maquetado estilístico para la revista } \\
\text { seleccionada. }\end{array}$ & $\mathbf{x}$ & $\mathbf{x}$ & $\vee$ \\
\hline
\end{tabular}




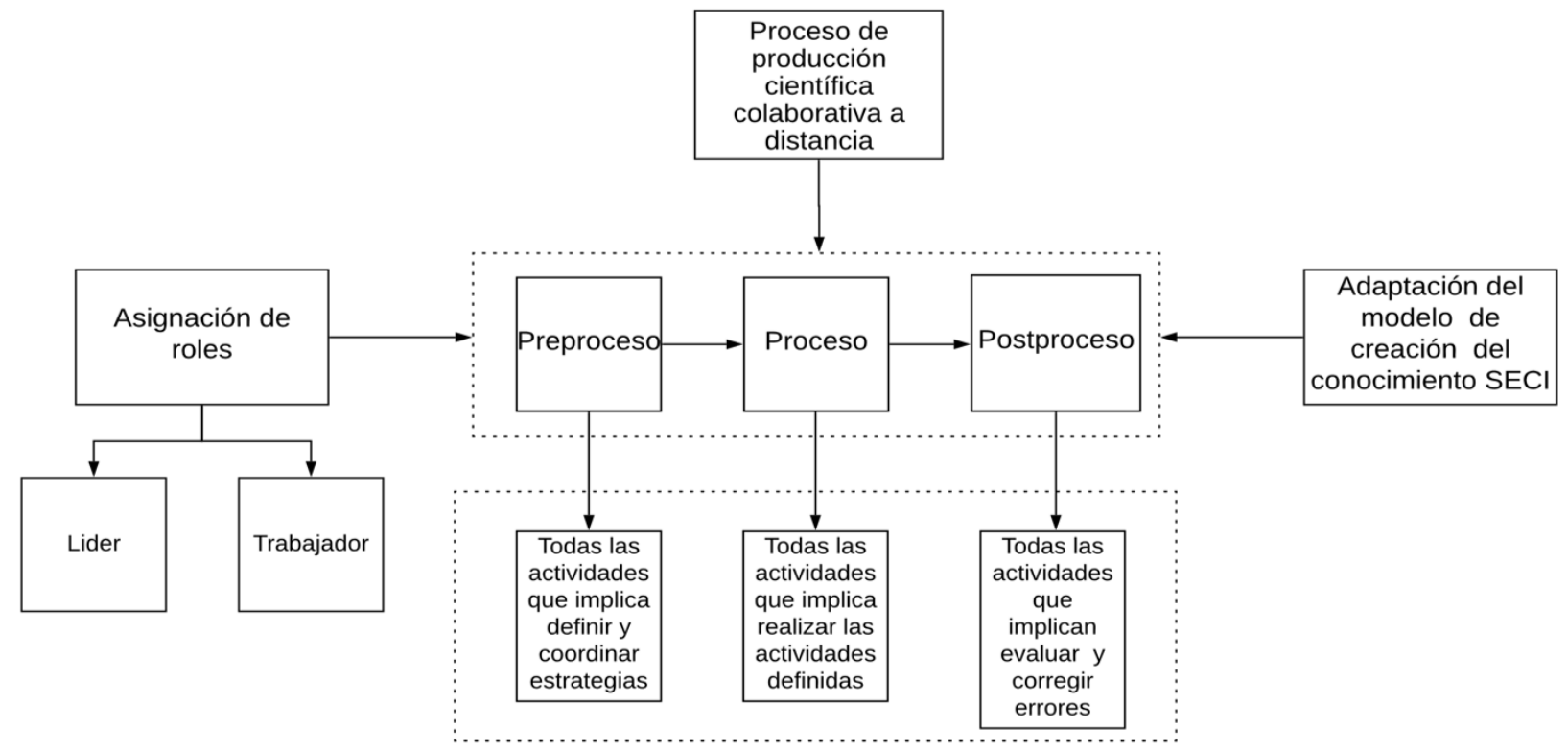

Figura 1. Modelo de escritura colaborativa a distancia. Fuente: elaboración propia.

\subsection{Modelado de las actividades colaborativas con thinklets}

Toda actividad que se desee modelar colaborativamente debe cumplir con tres criterios fundamentales: igual participación (IP), responsabilidad individual (RI) e interdependencia positiva (IPO). Para el modelo propuesto, la estrategia de trabajo es la siguiente: todos los participantes deben estar vinculados o conectados en un medio virtual y cada uno tiene un espacio de tiempo de 5 minutos para hacer su contribución. Al final los temas y subtemas son elegidos por votación (IPO). Ahora bien, cada participante debe preparar una temática y defenderla en el lapso mencionado, junto con obligaciones como ser puntual y participar activamente de la reunión, de lo contrario no se puede realizar la actividad. Se debe acordar una fecha para celebrar las reuniones sin faltas de los participantes, luego el líder procede a iniciar la reunión si todos están conectados, de lo contrario la cancela y se reprograma (RI) (IP). Este proceso (Tabla 2) es solo un ejemplo de cómo las actividades filtradas anteriormente pueden modelarse con los criterios anteriores.

\subsection{División de las actividades en fases de la metodología propuesta}

Una vez ejecutada la filtración de las actividades propuestas y el modelamiento de cada una de ellas, se procede a fraccionar las resultantes en tres fases principales: preproceso, proceso y postproceso, según la ejecución temporal en la que deben realizarse.

La fase de preproceso contiene todas aquellas actividades que tienen que ver con coordinar y definir las metodologías a utilizar para la investigación y escritura del artículo científico.

Esta fase es sumamente importante, ya que se realiza la planificación, definición y limitación de las actividades a realizar dentro de éstas. Las actividades propuestas en esta fase son procesos fundamentales para el éxito en la actividad colaborativa, pues definen gran parte de lo que se va a realizar en otras fases. 
Tabla 2. Ejemplo de modelamiento de actividades colaborativa con thinklets. Fuente: elaboración propia.

\begin{tabular}{|c|c|c|c|c|}
\hline Actividades & $\begin{array}{c}\text { Patrón } \\
\text { colaborativo }\end{array}$ & Thinklets & Justificación & $\begin{array}{c}\text { Awareness (Espacio de } \\
\text { trabajo) }\end{array}$ \\
\hline $\begin{array}{l}\text { Definir temática } \\
\text { principal del } \\
\text { artículo y } \\
\text { subtemas que } \\
\text { pueden aportar } \\
\text { a resolver la } \\
\text { problemática. }\end{array}$ & Reducción & $\begin{array}{l}\text { Discussion } \\
\text { Circle }\end{array}$ & $\begin{array}{l}\text { Este thinklet se basa en la técnica } \\
\text { del aprendizaje colaborativo } \\
\text { Inside-Outside Circle, que permite } \\
\text { a un grupo de personas } \\
\text { intercambiar ideas con la } \\
\text { participación de todos. Para ello, } \\
\text { los individuos se agrupan en } \\
\text { parejas para discutir un tema, } \\
\text { luego existe un intercambio de } \\
\text { parejas y se sigue la discusión } \\
\text { hasta el final de la rotación. }\end{array}$ & $\begin{array}{l}\text { Las actividades están } \\
\text { vinculadas a múltiples } \\
\text { espacios de trabajo } \\
\text { virtuales, que el líder } \\
\text { debe coordinar y } \\
\text { sincronizar; no obstante, } \\
\text { cada participante es } \\
\text { responsable de mantener } \\
\text { el progreso de su trabajo } \\
\text { para hacer visible su }\end{array}$ \\
\hline $\begin{array}{l}\text { Definir } \\
\text { metodología que } \\
\text { se va a utilizar } \\
\text { para resolver la } \\
\text { problemática. }\end{array}$ & & $\begin{array}{l}\text { Discussion } \\
\text { Chips }\end{array}$ & $\begin{array}{l}\text { En este Thinklet thinklet se } \\
\text { propone que se intervenga por } \\
\text { turnos con un elemento que } \\
\text { identifique al participante; luego, } \\
\text { este elemento deja de ser visible y } \\
\text { se reinicia el proceso si es } \\
\text { necesario. }\end{array}$ & $\begin{array}{l}\text { contribuir al } \\
\text { cumplimiento de los } \\
\text { objetivos. Los espacios de } \\
\text { trabajo escogidos por } \\
\text { consenso en las primeras } \\
\text { actividades del } \\
\text { preproceso, deberán }\end{array}$ \\
\hline $\begin{array}{l}\text { Búsqueda de } \\
\text { antecedentes } \\
\text { relacionados con } \\
\text { el fenómeno } \\
\text { estudiado. }\end{array}$ & Evaluación & $\begin{array}{l}\text { Analysis } \\
\text { Content }\end{array}$ & $\begin{array}{l}\text { El thinklet se basa en la técnica de } \\
\text { análisis de contenido que es muy } \\
\text { útil para la reducción de datos. } \\
\text { Cada participante se } \\
\text { comprometerá a analizar la } \\
\text { información sobre un tema. Para } \\
\text { ello, un tema se divide en } \\
\text { diferentes subtemas que lo } \\
\text { clarifiquen asignándole un } \\
\text { participante. Finalmente, se rotan } \\
\text { los contenidos y se crea un } \\
\text { conocimiento compartido. }\end{array}$ & $\begin{array}{l}\text { contribuir en dar } \\
\text { respuesta a los siguientes } \\
\text { interrogantes: ¿cuáles son } \\
\text { los otros miembros del } \\
\text { grupo y qué actividades } \\
\text { realizan?, ¿dónde están?, } \\
\text { ¿qué están haciendo?, } \\
\text { ¿qué han hecho ya?, ¿qué } \\
\text { van a hacer ahora? y } \\
\text { ¿cómo puedo ayudar a } \\
\text { otros para que completen } \\
\text { una actividad? }\end{array}$ \\
\hline
\end{tabular}

Ahora bien, la fase de proceso se compone de todas las actividades correspondientes a la realización de la producción científica. Es importante mencionar que cada una de ellas produce un activo que es utilizado para las siguientes actividades. La fase de proceso inicia una vez se realiza la recolección de información hasta que los conocimientos son cristalizados en un documento; por lo general es el conjunto de actividades que más conlleva tiempo y es donde se realiza la mayor interacción colaborativa entre los participantes, porque son actividades iterativas y de constante retroalimentación.

Finalmente, está el postproceso que corresponde a todas aquellas actividades que dan cierre a la investigación. En esta fase se reciben generalmente todos los insumos obtenidos de las fases anteriores. Aunque hay que aclarar que es sumamente importante, porque es aquí donde se realizan las correcciones sugeridas por los pares académicos, que es un momento importante para la correcta publicación del documento.

Una vez obtenidas todas las actividades que comprenden cada una de las tres fases para escribir artículos colaborativos (Tabla 3), se define qué roles debe cumplir cada persona involucrada en la actividad. Asimismo, se explica cómo influye el modelo SECI en este proceso.

Los roles de usuario para el modelo propuesto están relacionados con la generación de interdependencia positiva entre los participantes, de modo que las partes se sientan en la obligación de trabajar e involucrarse en la actividad. Por este motivo, se han definido dos tipos 
de roles los cuales son iterativos y están relacionados durante todas las fases del modelo: el primero denominado "líder" y el segundo "trabajador".

Tabla 3. Actividades que componen el proceso de escritura científica a distancia desde la virtualidad Fuente: elaboración propia.

\begin{tabular}{|c|c|c|}
\hline Actividades del preproceso & Actividades del proceso & $\begin{array}{l}\text { Actividades del } \\
\text { postproceso }\end{array}$ \\
\hline $\begin{array}{l}\text { Definir temática principal del artículo y subtemas } \\
\text { que pueden aportar a resolver la problemática. } \\
\text { Definir viabilidad del estudio } \\
\text { Lluvia de ideas sobre posibles soluciones para la } \\
\text { problemática propuesta. } \\
\text { Socializar la problemática con los otros autores, } \\
\text { cuestiones acerca de esta o del fenómeno de } \\
\text { estudio. } \\
\text { Asignar roles a los participantes. } \\
\text { Definir fechas de avances y entrega final. } \\
\text { Definir metodología que se va a utilizar para } \\
\text { resolver la problemática. } \\
\text { Definición del tipo de producción científica a } \\
\text { realizar (artículo científico, documento de } \\
\text { conferencia, proyecto, entre otros). } \\
\text { Definir medios de comunicación entre los autores. } \\
\text { Definición de la forma de presentar los resultados } \\
\text { específicos del artículo científico. }\end{array}$ & $\begin{array}{l}\text { Desarrollo de la metodología } \\
\text { propuesta. } \\
\text { Búsqueda de antecedentes } \\
\text { relacionados con el fenómeno } \\
\text { estudiado. } \\
\text { Escritura del artículo científico. } \\
\text { Realización del proceso de } \\
\text { experimentación o estudio de caso } \\
\text { (si es necesario). } \\
\text { Obtención y selección de resultados } \\
\text { relevantes. } \\
\text { Reestructuraciones o correcciones al } \\
\text { artículo científico o las metodologías } \\
\text { propuestas. } \\
\text { Aplicación de una estrategia o } \\
\text { técnica estadística a los resultados. } \\
\text { Definición de conclusiones o aportes } \\
\text { principales del trabajo. }\end{array}$ & $\begin{array}{l}\text { Realización de } \\
\text { correcciones y } \\
\text { comentarios de los } \\
\text { pares académicos. } \\
\text { Definición de } \\
\text { estrategia para } \\
\text { corrección o } \\
\text { sugerencias por } \\
\text { parte de los } \\
\text { evaluadores. } \\
\text { Proceso de } \\
\text { maquetado } \\
\text { estilístico para la } \\
\text { revista } \\
\text { seleccionada. }\end{array}$ \\
\hline
\end{tabular}

Hay que mencionar que estos roles son intercambiables y pueden ir rotando incluso en una misma actividad. La dinámica de estos es la siguiente: el líder es aquel que tiene más conocimiento sobre el tema o tiene la idea de cómo realizar la actividad colaborativa, mientras que los trabajadores siguen las directrices de éste y pueden aportar ideas para resolver la actividad, pero al final el líder debe tomar la decisión de cuál es la mejor forma de resolver o transitar la actividad.

Un ejemplo de la dinámica de los roles propuestos es la siguiente: la actividad es "definir temática principal del artículo y subtemas que pueden aportar a resolver la problemática". En este sentido el líder de esta actividad debe ser aquel que tuvo la idea o encontró la problemática, porque es quien tiene más experiencia y conocimientos sobre el campo a trabajar en el documento. El líder debe definir cuál será la temática principal, mientras que los trabajadores pueden aportar soluciones o alternativas en forma de sugerencias para ser tenidas en cuenta. Ahora, una vez determinada la dinámica de los roles mencionados, se procede a observar cómo el modelo SECI permite el crecimiento del conocimiento uniformemente.

\subsection{Implementación del modelo SECI en la propuesta}

Las actividades que se seleccionaron mediante la encuesta tienen dos características principales: en primer lugar, se pueden modelar colaborativamente, y en segundo, se utiliza alguna forma de transmisión del conocimiento al realizarlas (socialización, externalización, combinación o internalización) [9]; por esto es importante mencionar que la metodología propuesta tiene implícitamente las formas de transmisión propuestas en el modelo SECI; de esta forma el conocimiento se transfiere de manera uniforme entre los autores del documento científico.

En la Tabla 4, se describen las formas de transmisión del conocimiento utilizando cada una de las actividades propuestas para la producción de artículos de investigación de forma 
colaborativa. Basados en la evidencia de la tabla anterior, puede afirmarse que el modelo SECI está integrado en las actividades colaborativas para realizar un escrito científico desde la distancia; asimismo, para reforzar la integración del modelo en la metodología propuestas, se brinda una serie de sugerencias a la hora realizar las actividades colaborativas propuestas:

Todas las evidencias (insumos para otras actividades) de las actividades colaborativas deben estar accesibles a los autores, esto con el fin de incitar la interdependencia positiva entre los participantes, pues cada persona tendrá las mismas herramientas para trabajar en sus respectivas tareas.

Las herramientas de colaboración tales como procesadores de texto, códigos de fuente, presentaciones o gestores de referencias deben ser accesibles para todos.

Existe un espacio en común en el que los participantes pueden interactuar libremente.

Pueden preguntar o responder cuestiones acerca de la actividad colaborativa: a su vez es un espacio en que la comunicación debe ser rápida, eficaz y clara para que de esta forma no se pierda el momentum de la colaboración. Por ejemplo, esto puede ser un chat grupal mediante aplicaciones de mensajería instantánea.

Se mencionó anteriormente que el modelo SECI también plantea la existencia de un lugar donde las personas interactúan para que el conocimiento fluya. En este caso el concepto de lugar o "Ba" se adapta a la virtualidad y se convierte en el lugar donde los autores interactúan, remarcando que no solamente está supeditado a una aplicación de mensajería instantánea, también puede ser cualquier procesador de texto en línea, herramientas para organización de proyectos o servicios de alojamiento de archivos; es decir, cualquier instrumento que propicie la existencia de interacción entre los distintos participantes.

Tabla 4. Ejemplos de formas de comunicación según el modelo SECI en las actividades colaborativas propuestas Fuente: elaboración propia.

\begin{tabular}{|c|c|}
\hline Actividades & $\begin{array}{l}\text { Forma de transmisión del } \\
\text { conocimiento }\end{array}$ \\
\hline $\begin{array}{l}\text { Definir temática principal del artículo y subtemas que pueden aportar a } \\
\text { resolver la problemática. }\end{array}$ & Socialización \\
\hline Definir metodología que se va a utilizar para resolver la problemática. & Socialización \\
\hline Asignar roles a los participantes. & Socialización \\
\hline Definir fechas de avances y entrega final. & Externalización \\
\hline $\begin{array}{l}\text { Socializar la problemática con los otros autores, cuestiones acerca de esta o } \\
\text { del fenómeno de estudio, entre otros. }\end{array}$ & Socialización y Externalización \\
\hline Definir medios de comunicación entre los autores. & Socialización \\
\hline Búsqueda de antecedentes relacionados con el fenómeno estudiado. & Combinación \\
\hline Escritura del artículo científico. & Combinación e Internalización \\
\hline $\begin{array}{l}\text { Realización del proceso de experimentación o estudio de caso (si es } \\
\text { necesario). }\end{array}$ & Internalización \\
\hline Obtención y selección de resultados relevantes. & Socialización \\
\hline Aplicación de una estrategia a técnica estadística a los resultados. & Socialización \\
\hline Definición de conclusiones os aportes principales del trabajo. & Socialización \\
\hline Realización de las correcciones y comentarios de los pares académicos. & Externalización \\
\hline $\begin{array}{l}\text { Definición de estrategia para corrección o sugerencias por parte de los } \\
\text { evaluadores. }\end{array}$ & Externalización \\
\hline
\end{tabular}

Este proceso es fundamental debido a que, entre más interacción exista entre los miembros del equipo, generará más interdependencia positiva y responsabilidad individual, ocasionando el aumento de la probabilidad de éxito de la actividad colaborativa en cuestión. 


\subsection{Caso de estudio}

El modelo propuesto se implementó en un grupo de seis (6) investigadores pertenecientes al grupo de investigación Logiciel de la Fundación Universitaria de Popayán. Se les pidió redactar un artículo de investigación que consta de distintos componentes (título, introducción, planteamiento del problema, referentes teóricos, metodología, resultados, bibliografía); asimismo, se les socializó la metodología propuesta en una sección virtual en donde se respondieron preguntas acerca de está. Por último, se les comunicó acerca de una serie de herramientas en línea (WhatsApp, Trello, Parsifal, Google Docs, OneDrive y Zotero) y su funcionamiento para que se pueda implementar el modelo de manera más natural.

En un primer momento se estableció como "líder" al director del grupo de investigación, ya que es la persona que tenía la idea sobre la problemática a resolver con la propuesta de investigación. El líder estableció contacto con los participantes socializando la dinámica del trabajo en el contexto colaborativo. Adicionalmente, es importante mencionar que el rol se fue variando según el momento en que se encontraba el proceso investigativo; por ejemplo, otra persona tomó el rol de líder cuando se procedió a documentar sobre las investigaciones existentes, pues este individuo tenía experiencia utilizando la herramienta de apoyo a revisiones bibliográficas (Parsifal). Entre los elementos a destacar en el proceso de experimentación se puede mencionar que en total se tuvieron 8 sesiones conjuntas (todos los participantes), mediante la herramienta Google Meet, y 24 sesiones (algunos participantes) para intercambiar y discutir entregables para las actividades remotas. El promedio de las sesiones conjuntas fue de 30 minutos, mientras que las secciones entre algunos participantes tuvieron un tiempo aproximado de 18 minutos. También se estableció un chat grupal de mensajería instantánea para poder debatir ideas de forma rápida sobre la investigación.

La realización del documento se hizo en la herramienta en línea Google Docs, utilizando control de cambios para cada contribuidor. Igualmente se utilizó Trello para vigilar estrictamente las actividades realizadas en cada una de las fases, asegurando su ejecución en tiempo y forma. En la Figura 2 se pueden observar algunas evidencias del trabajo mencionado anteriormente. El equipo tardó cuatro semanas en realizar colaborativamente la actividad de escritura de una propuesta de investigación y al finalizar la actividad se hizo una encuesta para conocer el nivel de satisfacción de los participantes con el modelo colaborativo GCCWrite.

Investigación Fundación Universitaria Popayan में की 0

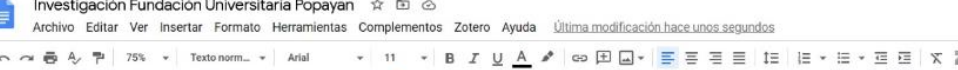

目

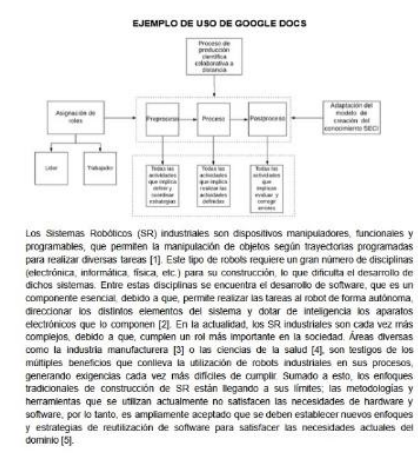

Figura 2. Ejemplo de espacios colaborativos utilizados para el modelo propuesto Fuente: elaboración propia.
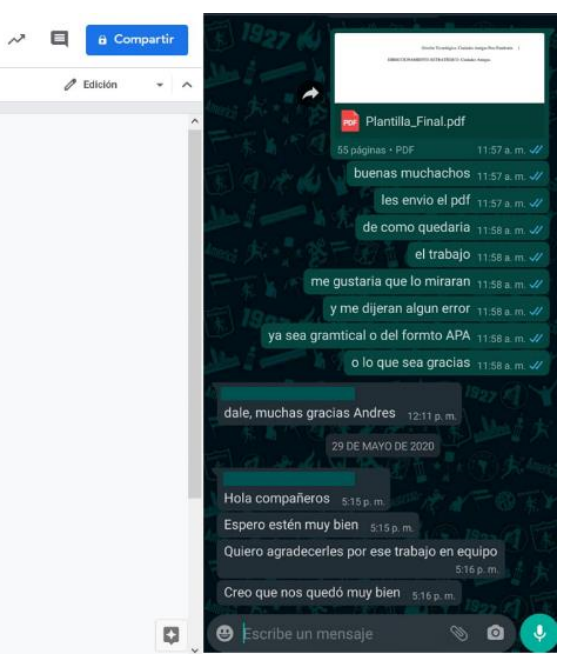


\section{RESULTADOS Y DISCUSIÓN}

A continuación, se presentan los resultados obtenidos durante el desarrollo de la investigación. En primer lugar, se expondrán aquellas evidencias encontradas en la encuesta realizada a los investigadores, los cuales arrojaron diferentes opiniones acerca del proceso investigativo en épocas de pandemia. Posteriormente, se expondrán los resultados obtenidos del caso de estudio realizado.

En cuanto a la encuesta se puede mencionar que ningún investigador utilizó un modelo, metodología o guía que les permita trabajar desde la distancia de forma colaborativa.

Asimismo, indicaron que lo más parecido a esto son las herramientas en línea como los chats grupales, correos electrónicos o procesadores de texto; sin embargo, aludieron que es importante desarrollar una manera sistemática que permita organizar este proceso (trabajo colaborativo a distancia para la producción científica), el cual ha cobrado relevancia por las condiciones actuales del mundo. Además, expresaron que existen ciertas dificultades a la hora de trabajar en casa, debido a que hay inconvenientes y limitaciones que no propician la colaboración, siendo la falta de compromiso de los participantes la causa principal del fracaso de las actividades colaborativas, esto suele ocurrir por la falta de interdependencia positiva y compromiso que se asume cuando se trabaja desde la virtualidad, por lo tanto, mencionan que este es un tema para resolver. También mencionaron que no existe una estrategia de seguimiento al trabajo de los diferentes participantes, lo que genera confusión entre las partes y retrabajo en ciertas áreas. Una opinión que fue común entre los encuestados es que es fundamental desarrollar una metodología que permita estructurar el proceso de producción científica garantizando aspectos como la igual participación, interdependencia positiva, compromiso y homogeneidad en el conocimiento. Por último, mencionaron que las herramientas virtuales actualmente son suficientes para realizar producción científica a distancia, pero el proceso de colaboración falla porque no es estructurado, generando errores en aspectos como la coordinación de los tiempos de trabajo.

El caso de estudio y la encuesta permitieron capturar información sobre el grado de satisfacción de los participantes con la metodología propuesta, permitiendo establecer un precedente para que esta sea replicada con actividades grupales del mismo tipo. Además, se analizaron datos sobre la frecuencia de las reuniones o la participación en chats grupales, lo que permite establecer valores aproximados para determinar el nivel de interdependencia positiva, la responsabilidad individual y el compromiso con la actividad.

En esta línea, la encuesta de satisfacción aplicada al grupo experimental arrojó como principal resultado un porcentaje alto de aceptación con la metodología propuesta, concretamente se mencionó que al ser un proceso estructurado permite una mejor coordinación de las actividades propuestas, facilitando la colaboración en cada fase. Respecto a la inclusión de roles en la metodología, los encuestados mencionaron un buen nivel de aceptación. Entre los aspectos positivos expresaron que este formato permite establecer una estructura organizacional ordenada, generando así una disposición organizada en las actividades y procesos que apoyan el objetivo final. En conclusión, indicaron que la inclusión del modelo SECI es un aporte beneficioso en el proceso de producción científica que puede ayudar a que el conocimiento sea homogéneo entre los participantes y de esta forma obtener mejores resultados investigativos.

Ahora bien, en lo que respecta a la medición de la colaboración entre los participantes se utilizó la propuesta de Casanova et al., en [41], donde se emplea el conteo de frases determinadas para establecer el porcentaje de colaboración en una actividad de cada una de las partes; para ello se consideró el número de veces que una persona menciona determinadas frases (descritas más abajo), ya que éstas se pueden asociar con un apartado específico de la 
colaboración. Los datos fueron recogidos del chat grupal, de las reuniones virtuales o por cualquier medio de interacción entre los participantes. En la Tabla 3 se puede observar el porcentaje de participación en tres apartados que permiten medir el proceso colaborativo con la metodología propuesta. A continuación, se define cada apartado.

Interdependencia positiva: la medición de este apartado se realiza mediante el conteo de frases tales como: "yo puedo trabajar en este apartado" o "me comprometo a realizar esto"; es decir, todas aquellas expresiones que tengan como fin aportar al objetivo común. Responsabilidad individual: la estimación de este apartado se realizó enumerando frases como: "envío el desarrollo de mi actividad" o "el día de mañana me comprometo a realizar esto"; es decir, son todas aquellas frases que denotan compromiso para realizar las actividades asignadas.

Igual participación: en este sentido se busca encontrar todas aquellas oraciones que indican trabajo colaborativo igualitario; por ejemplo: "te corresponde esta actividad" o "podemos trabajar juntos en".

Además, se debe aclarar que por cada frase de las anteriormente descritas se les asignaba un punto a los participantes. En la Tabla 5 se puede apreciar los resultados tabulados.

Tabla 5. Puntuaciones de los participantes con el modelo propuesto. Fuente: elaboración propia.

\begin{tabular}{lccc}
\hline Participantes & Interdependencia positiva & Responsabilidad individual & Igual participación \\
\hline Sujeto A & 20 & 7 & 8 \\
Sujeto B & 21 & 8 & 9 \\
Sujeto C & 15 & 5 & 10 \\
Sujeto D & 18 & 6 & 5 \\
Sujeto E & 22 & 8 & 6 \\
Sujeto F & 16 & 4 & 7 \\
\hline
\end{tabular}

Respecto a los resultados anteriores se puede mencionar que la metodología propuesta presenta una estricta interdependencia positiva entre los participantes. En general, los investigadores entendieron que el éxito individual contribuye al éxito grupal, y que el trabajo realizado por una persona es sumamente importante para alcanzar los objetivos de todos, por lo tanto, realizaron sus tareas en tiempo y forma, además de participar activamente en las reuniones, contribuyendo a mejorar el proceso de la actividad colaborativa. Asimismo, gracias a que el modelo permitió desarrollar las actividades según su ejecución temporal, esto provocó en los protagonistas la necesidad de finalizar sus actividades para que el resto de las personas pudieran realizar sus tareas, creando IPO entre ellos. El uso de roles permitió afianzar la interdependencia positiva, debido a que al existir un sistema de jerarquía y participación se invita a las partes a interactuar entre sí para solucionar la actividad general [42].

Respecto a la responsabilidad individual, se constató que la metodología ínsita a esta cualidad, pues permite que se distribuyan las tareas de forma uniforme, fomentando así el compromiso individual que aporta al objetivo común. Sumado a esto, la división en fases de la actividad colaborativa permite desarrollar estrategias de igual participación, como pequeñas evaluaciones en cada fase desarrollada (por ejemplo, en preproceso preguntar acerca de la literatura encontrada; en proceso cuestionarse acerca del estado de desarrollo de las actividades asignadas; y en el postproceso, un indicador podría ser entregar en tiempo y forma las asignaciones). Anteriormente se mencionó que las actividades se realizan según su ejecución temporal, lo que generó que los participantes estén constantemente involucrados en 
el proyecto, puesto que el retraso en una de las tareas puede ocasionar demora general en el proyecto. Esto se verificó en el caso de estudio, pues no se tuvieron retrasos en tiempos de entrega entre los individuos, lo que sugiere un alto nivel de compromiso de los participantes, más allá de casos puntuales en donde algunas partes tuvieron que recurrir a la ayuda de otros para terminar su actividad a tiempo.

En relación con la igual participación, se constató que el modelo propuesto permite la proliferación de este valor, pues estrategias como la asignación de roles rotativos, la división de tareas para que sean realizadas por varias personas y la introducción de un moderador (líder) son elementos ampliamente aceptados para ayudar y propiciar la participación igualitaria [43].

Aun así, hay que mencionar que si bien el modelo propuesto permite la flexibilidad en la utilización de herramientas de comunicación y de trabajo, se recomienda utilizar una serie de instrumentos que aseguren la colaboración de forma eficaz. En primer lugar, un medio de comunicación sincrónica que permita la interacción entre los individuos participantes en tiempo real, específicamente aplicaciones de videoconferencia como Google Meet, Skype o Zoom, por mencionar algunas. Este tipo de herramientas constituyen la vía principal de participación de las personas, por lo cual tiene una importancia fundamental. También se aconseja la utilización de un medio de interacción asíncrono (mensajería instantánea como WhatsApp o Telegram) que permite realizar aportes en tiempos distintos a los acordados, esto con el fin de que no se pierda el momentum de la colaboración durante la actividad. El uso de un instrumento compartido que permita la cristalización del conocimiento es esencial, debido a que permitirá transformar ideas intangibles en resultados palpables. En este caso, un procesador de texto como Google Docs u Overleaf son buenas alternativas. Finalmente, herramientas menos trascendentes como espacios para compartir archivos accesibles para todos (Google Drive o OneDrive), o software para lograr un control sobre las actividades del proyecto (Trello) son altamente recomendables también.

Por último se debe mencionar que una de las ventajas encontradas cuando se utilizó el modelo fue el aumento en la responsabilidad individual de los participantes, ya que la dinámica intrínseca de la propuesta promueve el trabajo igualitario y responsable entre las partes. Del mismo modo se observó que el modelo permite un control estricto sobre los productos resultantes en cada una de las actividades, lo que posibilita asegurar la calidad de los resultados finales. Posteriormente se detectó que, al estructurar el proceso de escritura científica a distancia, proporcionó a las personas un punto de partida sobre el cual trabajar, lo que ahorra tiempo en organizar dinámicas de actividades grupales.

Ahora bien, la principal debilidad encontrada en el modelo tiene que ver con la adaptación y comprensión de la propuesta, pues elementos como el uso de roles o la sincronización constante en el desarrollo del trabajo son factores para tener en cuenta. De igual forma, el uso de herramientas tecnológicas conlleva una curva de aprendizaje qué es importante desarrollar.

\section{CONCLUSIONES Y TRABAJOS FUTUROS}

En el presente documento se expuso una metodología para realizar producción científica de forma colaborativa y a distancia. En principio. la propuesta se dividió en tres fases principales y se agregaron elementos del modelo SECI para permitir que el conocimiento entre los autores sea homogéneo. También se propuso una dinámica de roles en la propuesta metodológica. El modelo fue probado mediante un caso de estudio donde se encontró que la metodología es un aporte importante para el trabajo colaborativo, propiciando un aumento en 
indicadores de éxito de actividades colaborativas, como la responsabilidad individual, la igual participación y la interdependencia entre los participantes. En esta línea, los resultados sugieren que el modelo propuesto puede contribuir a mejorar el proceso de escritura colaborativa a distancia; por lo tanto, si es aplicado en escenarios similares ayudaría a optimizar la actividad investigativa de forma general.

Actualmente, las Tecnologías de la Información y la Comunicación (TIC) son herramientas que pueden ser de gran ayuda para el desarrollo de las actividades colaborativas a distancia. A diferencia de épocas anteriores donde la madurez tecnológica de estas no era suficiente, hoy en día se tienen todas las condiciones para desarrollar un trabajo estructurado, organizado y eficaz para producir investigación científica dejando de lado las barreras geográficas. Sin embargo, lo anterior no solo se refiere a la escritura colaborativa, también el aprendizaje se ha beneficiado del desarrollo tecnológico. Es por esto por lo que es un momento crítico donde los investigadores deben invertir esfuerzos en desarrollar metodologías y herramientas que beneficien el trabajo colaborativo a distancia.

Entre los principales trabajos futuros se puede mencionar el desarrollo de un software independiente que permita estimar el nivel de participación en las actividades colaborativas con entornos virtuales, para que de esta forma se pueda automatizar el proceso de medición de indicadores de colaboración. En esta línea, otro trabajo que se podría desarrollar son nuevos indicadores para determinar la calidad del proceso de escritura colaborativa a distancia, enfocándose en aspectos como calidad de las aportaciones, o incluso, en la actividad colaborativa en general.

El desarrollo de este trabajo está enmarcado dentro de las líneas investigativas del grupo IDIS de la Universidad del Cauca, específicamente dentro de la materia denominada Ingeniería de la Colaboración (dictada por el autor 3), en el que se incentiva la investigación en este campo. De esta forma, se encontró una problemática latente por las condiciones actuales de aislamiento en el que se encuentra la sociedad, donde se evidenció que no existe una forma estructurada de realizar producción científica a distancia, lo que ha generado retraso en este proceso. En este sentido, la idea surgió por parte de los tres autores dentro de la clase, en el marco de un debate amistoso sobre las consecuencias de la pandemia. Una vez identificada la idea, se realizó la búsqueda de antecedentes que permitió determinar si existían soluciones a la problemática detectada. La dinámica se desarrolló a través de búsquedas en bases de datos. Una vez definida la problemática, los tres autores conceptualizaron la propuesta que se iba a trabajar: los autores 1 y 2 intentaron determinar e incluir los elementos del modelo de gestión del conocimiento SECI, mientras que el autor 3 incluía elementos de la ingeniería de la colaboración para terminar de valorar la propuesta.

Posteriormente, se diseñó la encuesta para la recolección de datos. En este sentido los tres autores trabajaron en el diseño y aplicación de la prueba, compartiéndolo a través de redes de investigación y contactos académicos. Ahora, una vez determinado los resultados de la encuesta (tabulación autores 1 y 2, mientras autor 3 hizo la validación de los resultados), se procedió a realizar el estudio de caso, en donde se utilizó elementos del grupo Logiciel, En ese sentido el autor 2, que tiene contactos dentro de éste, facilitó la organización logística del caso.

Se realizó la socialización de la metodología por parte de los tres autores con los elementos del grupo de investigación mencionado. Como herramientas y medios se utilizaron Google Meet, WhatsApp y las distintas herramientas que se mencionan en el texto. Esta infraestructura está dada por los correos universitarios que se utilizan en las instituciones académicas. La validación y el análisis formal de los datos fue realizada por los tres autores para corroborar la veracidad de estos. Finalmente, la escritura estuvo a cargo de los autores 1 y 2, mientras la revisión, el direccionamiento y la supervisión estuvo a cargo del autor 3, el cual es considerado un experto en el campo de la ingeniería de la colaboración. 


\section{AGRADECIMIENTOS}

Los autores agradecen la asistencia financiera de la Red HCI-Collab y la Red VG-Collab.

\section{CONTRIBUCIONES}

Andrés Felipe Solis Pino: autor del tema a desarrollar, revisión de la literatura, desarrollador de la investigación en cuanto a la realización de pruebas experimentales, redacción inicial del manuscrito presentado y correcciones.

Leydi Mercedes Vargas Ordóñez: revisión de la literatura, desarrollador de la investigación en cuanto a la realización de pruebas experimentales, socialización del modelo propuesto, redacción inicial del manuscrito presentado.

César Alberto Collazos: conceptualización, direccionamiento del tema a desarrollar, seguimiento de la investigación, socialización del modelo propuesto y redacción del manuscrito y correcciones.

\section{CONFLICTOS DE INTERES}

No hay conflicto de intereses que declarar.

\section{REFERENCIAS}

[1] D. Biber; B. Gray, "Challenging stereotypes about academic writing: Complexity, elaboration, explicitness," J. Engl. Acad. Purp., vol. 9, no. 1, pp. 2-20. Mar. 2010. https://doi.org/10.1016/j.jeap.2010.01.001

[2] K. Hyland, "Academic publishing and the myth of linguistic injustice," J. Second Lang. Writ., vol. 31, pp. 58-69, Mar. 2016. https://doi.org/10.1016/j.jslw.2016.01.005

[3] V. Singh; P. Mayer, "Scientific writing: Strategies and tools for students and advisors: Strategies and Tools for Students and Advisors," Biochem. Mol. Biol. Educ., vol. 42, no. 5, pp. 405-413. Oct. 2014. https://doi.org/10.1002/bmb.20815

[4] D. Rodriguez; R. García-Martínez, "A Proposal of Interaction Modelling Formalisms in Virtual Collaborative Work Spaces," Lect. Notes Softw. Eng., vol. 2, no. 1, pp. 76-80. Feb. 2014. https://doi.org/10.7763/LNSE.2014.V2.98

[5] H. A. Rivera Rodríguez; M. N. Malaver Rojas, La organización: los stakeholders y la responsabilidad social, Editorial Universidad del Rosario. 2011. URL

[6] E. J. Agudelo Ceballos; A. Valencia Arias, "La gestión del conocimiento, una política organizational para la empresa de hoy," Ingeniare Rev. Chil. Ing., vol. 26, no. 4, 673-684. Dic. 2018. http://dx.doi.org/10.4067/S0718-33052018000400673

[7] H. Bathelt; A. Malmberg; P. Maskell, "Clusters and knowledge: local buzz, global pipelines and the process of knowledge creation," Prog. Hum. Geogr., vol. 28, no. 1, pp. 31-56. Feb. 2004. https://doi.org/10.1191/0309132504ph469oa

[8] G. von Krogh; J. Roos, Organizational epistemology. Macmillan, 1995.

[9] M. L. Farnese; B. Barbieri; A. Chirumbolo; G. Patriotta, "Managing Knowledge in Organizations: A Nonaka's SECI Model Operationalization," Front. Psychol., vol. 10, no. 2730, Dec. 2019. https://doi.org/10.3389/fpsyg.2019.02730

[10] M. Dávideková; J. Hvorecký, "Collaboration Tools for Virtual Teams in Terms of the SECI Model," en Interactive Collaborative Learning, Cham. 2017, vol. 544, pp. 97-111. https://doi.org/10.1007/978-3-31950337-0 9

[11] J. L. Jurado Muñoz; C. J. Pardo Calvache, "La gestión de proyectos Software, una prospectiva en la aplicación de estrategias en la Ingeniería colaborativa,” Lámpsakos, no. 9, 26-33. 2013. https://doi.org/10.21501/21454086.854 
[12] M. Coto; C. A. Collazos; S. M. Rivera, "Modelo Colaborativo y Ubicuo para apoyar los procesos de enseñanzaaprendizaje a nivel Iberoamericano," Rev. Educ. Distancia. no. 48. Mar. 2016. https://revistas.um.es/red/article/view/253521

[13] X. Cheng; X. Wang; J. Huang; A. Zarifis, "An Experimental Study of Satisfaction Response: Evaluation of Online Collaborative Learning," Int. Rev. Res. Open Distrib. Learn., vol. 17, no. 1, Feb. 2016. https://doi.org/10.19173/irrodl.v17i1.2110

[14] H. Lahti; P. Seitamaa-Hakkarainen; K. Hakkarainen, "Collaboration patterns in computer supported collaborative designing," Des. Stud., vol. 25, no. 4, 351-371. Jul. 2004. https://doi.org/10.1016/j.destud.2003.12.001

[15] R. Briggs; G. Kolfschoten; G-J. de Vreede; D. Douglas, "Defining key concepts for collaboration engineering," en AMCIS 2006 Proceedings, vol. 17, pp. 121-128. URL

[16] T. Giesbrecht; G. Schwabe; B. Schenk, "Service encounter thinklets: how to empower service agents to put value co-creation into practice: Service encounter thinklets," Inf. Syst. J., v. 27, no. 2, 171-196. Mar. 2017. https://doi.org/10.1111/isj.12099

[17] G. L. Kolfschoten; F. Niederman; R. O. Briggs; G.-J. De Vreede,"Facilitation roles and responsibilities for sustained collaboration support in organizations," J. Manag. Inf. Syst., v. 28, no. 4, 129-162. Dec. 2014. https://doi.org/10.2753/MIS0742-1222280406

[18] A. Solano Alegría; Y. Méndez Alegría; C. Collazos Ordóñez, "Thinklet: elemento clave en la generación de métodos colaborativos para evaluar usabilidad de software," Cienc. E Ing. Neogranadina, vol. 20, no. 2, pp. 87-106. https://doi.org/10.18359/rcin.278

[19] Y. A. Rodríguez Mora; J. Balladares-Burgos, “Aprendizaje colaborativo en entornos virtuales,” (Tesis de Maestría), Universidad Andina Simón Bolívar, Sede Ecuador, Quito, 2019.

[20] O. Revelo-Sánchez; C. A. Collazos-Ordóñez; J. A. Jiménez-Toledo. "El trabajo colaborativo como estrategia didáctica para la enseñanza/aprendizaje de la programación: una revisión sistemática de literatura," TecnoLógicas, vol. 21, no. 41, pp. 115-134. Jan. 2018. https://doi.org/10.22430/22565337.731

[21] J. Fernandez-Rio; J. A. Cecchini; A. Méndez-Giménez; D. Méndez-Alonso; J. A. Prieto. "Diseño y validación de un cuestionario de medición del aprendizaje cooperativo en contextos educativos," An. Psicol. Psychol., vol. 33, no. 3, pp. 680-688, Jul. 2017. https://doi.org/10.6018/analesps.33.3.251321

[22] G. M. Londoño M., "Aprendizaje colaborativo presencial, aprendizaje colaborativo mediado por computador e interacción: Aclaraciones, aportes y evidencias," Rev. Q., vol. 2, no. 4. 2008. URL

[23] B. Figueroa; M. Aillon, "Escritura académica de un ensayo mediado por el aprendizaje colaborativo virtual," Estud. Pedagógicos Valdivia, vol. 41, no. 1, pp. 79-91. 2015. http://dx.doi.org/10.4067/S071807052015000100005

[24] D. Rodríguez; R. Priano; N. Charczuk; R. García Martínez; R. García, "Medidas de interacción en espacios virtuales para trabajo colaborativo," en XVII Workshop de Investigadores en Ciencias de la Computación, Red de Universidades con Carreras en Informática, Salta, 2015. URL

[25] S. Álvarez Lebrum; O. M. Salazar; D. A. Ovalle, "Hacia un modelo ontológico de aprendizaje colaborativo basado en agentes". Rev. Vínculos., vol. 13, no. 1, pp. 45-55. Jun. 2016. https://doi.org/10.14483/2322939X.11581

[26] D. Rodríguez; R. Priano; R. García; N. Charczuk; F. Ribeiro; S. Bianco, "Programa de I+ D+ I en ingeniería de espacios virtuales de trabajo," en XVIII Workshop de Investigadores en Ciencias de la Computación, Entre Ríos, 975-977. URL

[27] S. Tyagi; S. Agrawal; K. Yang; H. Ying, "An extended Fuzzy-AHP approach to rank the influences of socialization-externalization-combination-internalization modes on the development phase," Appl. Soft Comput., vol. 52, 505-518. Mar. 2017. https://doi.org/10.1016/j.asoc.2016.10.017

[28] Y. Hijikata; T. Takenaka; Y. Kusumura; S. Nishida. "Interactive knowledge externalization and combination for SECI model," en Proceedings of the 4th international conference on Knowledge capture - KCAP '07, Whistler, BC, Canada. 2007, pp. 151-158. https://doi.org/10.1145/1298406.1298434

[29] A. Prasetya; K. Taroreh, "The Implementation of Socialization, Externalization, Combination, and Internalization (SECI) Through EDMODO Application to Improve Student Group's Learning Outcomes," en 1st International Conference on Education Innovation (ICEI 2017). Atlantis press, 2018, pp. 5-8. https://doi.org/10.2991/icei-17.2018.2

[30] L. A. Guerrero; B. Mejías; C. A. Collazos; J. A. Pino; S. F. Ochoa, "Collaborative learning and creative writing", en Proceedings of the IEEE/LEOS 3rd International Conference on Numerical Simulation of Semiconductor Optoelectronic Devices (IEEE Cat. No. 03EX726). Santiago, Chile, 2003, pp. $180-186$. http://dx.doi.org/10.1109/LAWEB.2003.1250295

[31] L. Ubilla Rosales; L. Gómez Álvarez; K. Sáez Carrillo, "Escritura colaborativa de textos argumentativos en inglés usando Google Drive,” Estud. Pedagógicos Valdivia, vol. 43, no. 1, pp. 331-348, 2017. http://dx.doi.org/10.4067/S0718-07052017000100019 
[32] Á. G. Santa-Cecilia. "Bases comunes para una Europa plurilingüe: Marco común europeo de referencia para las lenguas: aprendizaje, enseñanza, evaluación,” Tarbiya Rev. Investig. E Innov. Educ., no. 33. Feb. 2003. URL

[33] V. Ness; K. Duffy; J. McCallum; L. Price, "Getting published: Reflections of a collaborative writing group," Nurse Educ. Today, vol. 34, no. 1, pp. 1-5. Jan. 2014. https://doi.org/10.1016/j.nedt.2013.03.019

[34] D. Hasanuddin; E. Emzir; S. Akhadiah. "Improving Students' Scientific Writing Ability through Blended learning Based Collaborative Learning," Int. J. Emerg. Technol. Learn. (IJET), vol. 14, no. 20, pp. 34-43. 2019. https://doi.org/10.3991/ijet.v14i20.11457

[35] S. M. Aluisio; I. Barcelos; J. Sampaio; O. N. Oliveira. "How to learn the many unwritten "rules of the game" of the academic discourse: a hybrid approach based on critiques and cases to support scientific writing," en Proceedings IEEE International Conference on Advanced Learning Technologies. Madison, 2001, pp. 257260. https://doi.org/10.1109/ICALT.2001.943916

[36] C. Gamberi; K. Hall. "Undergraduates can publish too! A case study of a scientific team writing assignment leading to publication." Int. J. Sci. Educ., vol. 41, no. 1, pp. 48-63. Oct. 2018. https://doi.org/10.1080/09500693.2018.1531439

[37] I. D. Claros; C. A. Collazos; R. Cobos, "Servicios awareness para la mejora del escenario educativo soportado sobre KnowCat," Av. En Sist. E Informática, vol. 6, no. 1, pp. 127-134. Jan. 2009. URL

[38] C. A. Collazos, "Diseño de actividades de aprendizaje colaborativo asistidas por computador". Rev. Educ. En Ing., vol. 9, no. 17, pp. 143-149. Jun. 2014. https://doi.org/10.26507/rei.v9n17.379

[39] P. E. Glinz Férez, "Un acercamiento al trabajo colaborativo," Rev. Iberoam. Educ., vol. 36, no. 7, Feb. 2005. https://doi.org/10.35362/rie3672927

[40] M. Laal, "Positive Interdependence in Collaborative Learning," Procedia - Soc. Behav. Sci., vol. 93, pp. 14331437. Oct. 2013. https://doi.org/10.1016/j.sbspro.2013.10.058

[41] M. O. Casanova Uribe; I. M. Alvarez Valdivia; I. Gómez Alemany, "Propuesta de indicadores para evaluar y promover el aprendizaje cooperativo en un debate virtual," Edutec Rev. Electrónica Tecnol. Educ., no. 28, Mar. 2009. https://doi.org/10.21556/edutec.2009.28.455

[42] P. Saenz-López Buñuel, Educar Emocionando, Universidad de Huelva. 2020. URL

[43] C. Velasquez Callado, "Aprendizaje cooperativo: aproximación teórico-práctica aplicada a la educación física," EmásF Rev. Digit. Educ. Física, vol. 5, no. 29, pp. 19-31. Jul. 204. URL 